OPEN ACCESS

Check for updates

\title{
The effects of plasma exchange in patients with ANCA-associated vasculitis: an updated systematic review and meta-analysis
}

\author{
Michael Walsh, ${ }^{1,2,3}$ David Collister, ${ }^{3,4}$ Linan Zeng, ${ }^{2,5}$ Peter A Merkel, ${ }^{6}$ Charles D Pusey, ${ }^{7}$ \\ Gordon Guyatt, ${ }^{1,2}$ Chen Au Peh,, ${ }^{8,9}$ Wladimir Szpirt, ${ }^{10}$ Toshiko Ito-Hara, ${ }^{11,12}$ David R W Jayne, ${ }^{13}$ \\ on behalf of the Plasma exchange and glucocorticoid dosing for patients with ANCA-associated \\ vasculitis BMJ Rapid Recommendations Group*
}

For numbered affiliations see end of the article

*See supplementary material for collaborating authorship Correspondence to: M Walsh St Joseph's Hospital, Hamilton, ON, Canada L8N 4A6 lastwalsh1975@gmail.com (ORCID 0000-0001-8292-2014) Additional material is published online only. To view please visit the journal online.

Cite this as: BMJ 2022;376:e064604 http://dx.doi.org/10.1136/ bmj-2021-064604

Accepted: 16 December 2021

\section{ABSTRACT}

OBJECTIVE

To assess the effects of plasma exchange on important outcomes in anti-neutrophil cytoplasm antibody (ANCA)-associated vasculitis (AAV).

\section{DESIGN}

Systematic review and meta-analysis of randomised controlled trials.

\section{ELIGIBILITY CRITERIA}

Randomised controlled trials investigating effects of plasma exchange in patients with AAV or pauciimmune rapidly progressive glomerulonephritis and at least 12 months' follow-up.

\section{INFORMATION SOURCES}

Prior systematic reviews, updated by searching Medline, Embase, and CENTRAL to July 2020.

RISK OF BIAS

Reviewers independently identified studies, extracted data, and assessed the risk of bias using the Cochrane Risk of Bias tool.

\section{SYNTHESIS OF RESULTS}

Meta-analyses were conducted using random effects models to calculate risk ratios and $95 \%$ confidence intervals. Quality of evidence was summarised in accordance with GRADE methods. Outcomes were assessed after at least12 months of follow-up and included all-cause mortality, end stage kidney disease (ESKD), serious infections, disease relapse, serious adverse events, and quality of life.

RESULTS

Nine trials including 1060 participants met eligibility criteria. There were no important effects of plasma exchange on all-cause mortality (relative risk 0.90 ( $95 \% \mathrm{Cl} 0.64$ to 1.27), moderate certainty). Data from

\section{WHAT IS ALREADY KNOWN ON THIS TOPIC}

Plasma exchange has been used to treat some patients with anti-neutrophil cytoplasm antibody (ANCA)-associated vasculitis (AAV) since the 1980s on the basis of biological rationale and small clinical trials

\section{WHAT THIS STUDY ADDS}

This meta-analysis compiles the most recent and detailed data available from randomised controlled trials examining the effects of plasma exchange on important outcomes in patients with AAV

It found plasma exchange, when added to standard therapies, reduced the risk of end stage kidney disease at one year, regardless of baseline kidney function, and increased the risk of serious infections, a previously unrecognised effect Plasma exchange did not change the risk of death seven trials including 999 participants that reported ESKD demonstrated that plasma exchange reduced the risk of ESKD at 12 months (relative risk 0.62 (0.39 to 0.98 ), moderate certainty) with no evidence of subgroup effects. Data from four trials including 908 participants showed that plasma exchange increased the risk of serious infections at 12 months (relative risk 1.27 (1.08 to 1.49), moderate certainty). The effects of plasma exchange on other outcomes were uncertain or considered unimportant to patients.

\section{LIMITATIONS OF EVIDENCE}

There is a relative sparsity of events, and treatment effect estimates are therefore imprecise. Subgroup effects at the participant level could not be evaluated.

INTERPRETATION

For the treatment of AAV, plasma exchange has no important effect on mortality, reduces the 12 month risk of ESKD, but increases the risk of serious infections.

\section{FUNDING}

No funding was received.

\section{REGISTRATION}

This is an update of a previously unregistered systematic review and meta-analysis published in 2014.

\section{Introduction}

Granulomatosis with polyangiitis and microscopic polyangiitis are commonly grouped together as antineutrophil cytoplasm antibody (ANCA)-associated vasculitis (AAV). Due to the varied symptoms and organs involved when patients with AAV present, they are often cared for in hospital by several different specialties. Although treatment with immunosuppression and glucocorticoids improve the outcomes of patients with $\mathrm{AAV}$, they remain at high risk of death, end stage kidney disease (ESKD), and serious infections, particularly in the first year of treatment. ${ }^{12}$

Plasma exchange (PLEX) mechanically removes circulating plasma constituents, including immunoglobulins such as ANCA. The rapid removal of autoantibodies may reduce damage incurred by AAV and therefore reduce the risk of death and ESKD. However, the removal of antibodies, as well as the use of blood products and the potential need for central venous access, may also increase the risk of harms, particularly serious infections, the most common cause of death for patients with AAV. ${ }^{2}$

Given the publication of a large trial evaluating the effects of PLEX in patients with AAV (PEXIVAS), 
we updated a previous systematic review and metaanalysis of randomised control trials (RCTs) to better guide recommendations for the use of PLEX in AAV. ${ }^{3}$ This review is conducted as part of the BMJ Rapid Recommendations project, a collaborative initiative from the MAGIC Evidence Ecosystem Foundation (www.magicproject.org) and The BMJ. This systematic review informs a $B M J$ Rapid Recommendation addressing PLEX in addition to immunosuppression and glucocorticoid therapy in patients with severe AAV (see box 1). ${ }^{4}$

\section{Methods}

No ethical approval was required to conduct this metaanalysis.

\section{Search strategy \\ We previously searched Medline (1950 through August 2009), Embase (1980 through August 2009), and the Cochrane Controlled Clinical Trials Register (1996 through August 2009) using the Ovid search engine with two comprehensive search themes combined using the Boolean operator "and." 5 The first theme, vasculitis, was created using the Boolean search term "or" to search for the following terms, text words, and database-specific thesaurus terms: "vasculitis" or "ANCA" or "antibodies, antineutrophil cytoplasm" or "Wegener's granulomatosis" or "microscopic polyangiitis" or "microscopic polyarteritis" or "rapidly progressive glomerulonephritis" or "RPGN" or "pauci-immune glomerulonephritis" or "crescentic glomerulonephritis." The second theme, plasma exchange, was created to search for the text words and database-specific thesaurus terms "plasma exchange" or "plasmapheresis" or "apheresis." The results were filtered for randomised controlled trials. We added to the literature from this search by repeating the search}

\section{Box 1: Linked resources for this BMJ Rapid Recommendations cluster}

- Zeng L, Walsh M, Guyatt GH, et al. Plasma exchange and glucocorticoid dosing for patients with ANCA-associated vasculitis: a clinical practice guideline. BMJ 2022;376:e064597

- Summary of the results from the Rapid Recommendation process

- Walsh M, Collister D, Zeng L, et al. The effects of plasma exchange in patients with ANCA-associated vasculitis: an updated systematic review and meta-analysis. BMJ 2022;376:e064604

o Review and meta-analysis of randomised trials that assess effects of plasma exchange for antineutrophil cytoplasmic antibody (ANCA)-associated vasculitis (AAV)

- Xiao Y, Guyatt G, Zeng L, et al. The comparative efficacy and safety of alternative glucocorticoids regimens in patients with ANCA-associated vasculitis: a systematic review. BMJ Open 2022; doi:10.1136/bmjopen-2021-050507

- Review and meta-analysis of randomised trials that assess effects of alternative glucocorticoids regimens for AAV

- Walsh M. Predicting the 1-year risk of kidney failure in ANCA associated vasculitis. BMC Medicine (forthcoming)

- Prediction model of risk of kidney failure in AAV

- MAGICapp (https://app.magicapp.org/\#/guideline/4218)

o Expanded version of the results with multilayered recommendations, evidence summaries, and decision aids for use on all devices on literature from January 2009 to July 2020. We added the term "granulomatosis with polyangiitis" to the search string and used the "controlled trials, all" filter on Ovid. ${ }^{6}$

\section{Inclusion and exclusion criteria}

Two reviewers (MW and DC) independently evaluated all articles from both the first systematic review and the updated search for eligibility by first reviewing all identified abstracts. Abstracts that either evaluator did not identify as definitely ineligible underwent full text eligibility review. Inclusion criteria consisted of: (a) study design (RCT); (b) study population (patients with AAV or rapidly progressive glomerulonephritis of which $>75 \%$ of cases were considered pauci-immune or idiopathic); (c) the intervention (PLEX) by any method and in any dose as initial therapy in addition to immunosuppression and glucocorticoids); (d) the outcome (trials that report at least one of mortality, ESKD, serious infections, relapse of vasculitis, serious adverse events, or health related quality of life); and $(e)$ time (outcomes reported at 12 months after randomisation or later). Serious infections were defined as infections requiring hospitalisation or intravenous antibiotics. Studies were eligible whether published or unpublished and irrespective of language. Studies were excluded if they included primarily children $(>75 \%$ with age $<16$ years).

\section{Risk of bias assessment}

Two reviewers (MW and DC) assessed study quality independently using the Cochrane tool for assessing risk of bias that includes: random sequence generation; allocation concealment; blinding of participants, staff, and outcome assessors; incomplete outcome data; selective outcome reporting; and other important risks. ${ }^{7}$

\section{Data extraction}

For each eligible study, two reviewers independently extracted the following: study characteristics: population, description of intervention, and outcomes. Study outcomes were extracted for 12 months after randomisation and for longer term follow-up for each study and whenever possible for each pre-specified subgroup within a study (creatinine $<500 \mu \mathrm{mol} / \mathrm{L}$ $v \geq 500 \mu \mathrm{mol} / \mathrm{L}$ or on dialysis at baseline, no lung haemorrhage $v$ lung haemorrhage at baseline). Reviewers resolved disagreements through discussion.

\section{Certainty of evidence assessment}

We assessed certainty of evidence using the minimally contextualised GRADE approach (that is, we assessed the certainty of a minimally important effect being present). ${ }^{8}$ Minimally important differences were chosen by the Plasma exchange and glucocorticoid dosing for patients with ANCA-associated vasculitis BMJ Rapid Recommendations Group, including absolute risk differences of $2 \%$ for mortality, $3 \%$ for ESKD, and $5 \%$ for serious infections. ${ }^{4}$ We used commonly accepted thresholds for health-related 
quality of life instruments: 0.3 for the EQ-5D Index and 3 points for the EQ-5D health thermometer and SF36 physical and mental composite scores. Still using the minimally contextualised approach, for all other outcomes, we used the null effect as the threshold and rated the certainty whether there was an effect. Ratings of certainty included consideration of risk of bias, inconsistency, indirectness, and imprecision.

\section{Statistical analysis}

For each outcome, we used the random-effects model based on the DerSimonian and Laird method to estimate the average relative risk (RR) across trials and the corresponding 95\% confidence intervals (CI) corrected for uncertainty in between-study variance using the Hartung-Knapp method at 12 months and longer follow-up. ${ }^{910}$ We calculated the proportion of total variability in effect estimates due to betweenstudy heterogeneity (the $\mathrm{I}^{2}$ statistic) and estimated the between-study variance $\left(\tau^{2}\right)$. For studies with no events in one cell, we used a small continuity correction factor of 0.05 added to each cell of that study to reduce potential bias in estimates. ${ }^{11} 12$ We explored the possible effects of this correction factor using sensitivity analyses in which we omitted studies with no events in one group, used a continuity correction factor of 0.5 added to each cell and an empirical approach. ${ }^{12}$ We did not test for publication bias due to the low power of asymmetry tests in meta-analyses with small numbers of studies.

We explored subgroup effects on the basis of baseline kidney function by comparing subgroups with a creatinine concentration $\geq 500 \mu \mathrm{mol} / \mathrm{L}$ or receiving dialysis and subgroups with a creatinine concentration $<500 \mu \mathrm{mol} / \mathrm{L}$ and not receiving dialysis on the basis of previous studies concluding important effects of PLEX were limited to patients with a creatinine level $\geq 500 \mu \mathrm{mol} / \mathrm{L}$ or who were receiving dialysis. ${ }^{1314}$ We also explored subgroup effects on the basis of subgroups of patients with lung haemorrhage at baseline compared with those without lung haemorrhage (irrespective of kidney function) on the basis of recommendations that patients with lung haemorrhage receive particular benefit from PLEX. ${ }^{15}$ Subgroup effects were assessed by meta-analysis of only those trials that included patients in both subgroups to reduce the possible ecological biases by constructing multi-level random effects logistic regression models in which contributing trials were considered random factors and the treatment group-by-subgroup interactions and the corresponding interaction Pvalues were calculated. We performed sensitivity analyses of subgroup effects by also including trials that included patients in only one subgroup (for example, all patients had a creatinine level $\geq 500 \mu \mathrm{mol} / \mathrm{L}$ ). Statistical analyses were performed using Stata versions 16 (StatCorp, College Station, TX).

We examined the conclusiveness of our estimates with trial sequential analyses. We assumed the control event rate was the expected event rate for each outcome and assessed whether we had sufficient information to reliably conclude a treatment effect existed or did not exist with $90 \%$ power and a twosided $\alpha$ of 0.05 using O'Brien-Flemming $\alpha$ spending. ${ }^{16}$ We used Trial Sequential Analysis software (www.ctu. $\mathrm{dk} / \mathrm{tsa}$ ) for these analyses. We limited these analyses to the outcomes of all-cause mortality, ESKD, and serious infections, all at the time of one year. For trials that failed this test, we rated down certainty of evidence for imprecision.

We calculated absolute risk reductions by applying the point estimates of the relative risk and the boundaries of its $95 \%$ confidence interval to the absolute risk of the overall control groups included in each analysis. We recognised that the baseline risk of outcomes such as ESKD and serious infection vary substantially across the population of patients with AAV for which PLEX might be considered. We therefore calculated the absolute risk reductions across a range of baseline risks for outcomes with conclusive results. The range of risks was derived from the available literature of non-PLEX treated patients with AAV. Because these risks corresponded well with baseline serum creatinine concentration, we estimated baseline risks for four groups: low risk (creatinine $\leq 200 \mu \mathrm{mol} / \mathrm{L}$ ), low-moderate risk (creatinine $>200$ $300 \mu \mathrm{mol} / \mathrm{L}$ ), moderate-high risk (creatinine >300$500 \mu \mathrm{mol} / \mathrm{L}$ ), and high risk (creatinine $>500 \mu \mathrm{mol} / \mathrm{L}$ or requiring dialysis). ${ }^{17}$

\section{Patient and public involvement}

As per the BMJ Rapid Recommendations process, a guideline panel that included content experts, methodologists, and patients provided input into the review. The panel reviewed the population, selected and ranked important outcomes, subgroup analyses, minimally important differences, and recommended baseline risks on which absolute treatment effects were calculated. The panel decided to conduct analyses at one year of follow-up because it is commonly reported, a large proportion of events occur during this time, and it is short enough to reflect the effects of an acute treatment and be relevant to acute treatment decisions for patients and decision makers. The panel also decided to aggregate longer term followup since the epidemiological data suggested modest changes in risk of death, ESKD, and infection during broad follow-up periods after one year. This decision was reinforced by the relative lack of heterogeneity in meta-analyses of outcomes at longer term followup despite follow-up durations that varied between studies.

\section{Results}

The updated search identified 49 records in addition to studies from the previous meta-analysis. After screening titles and abstracts, five were identified for full text review, of which three papers not included in the previous meta-analysis proved eligible. ${ }^{31819}$ Two papers updated the results of previously included trials. ${ }^{1819}$ Of the nine previously included studies, one was excluded because it reported outcomes only at 


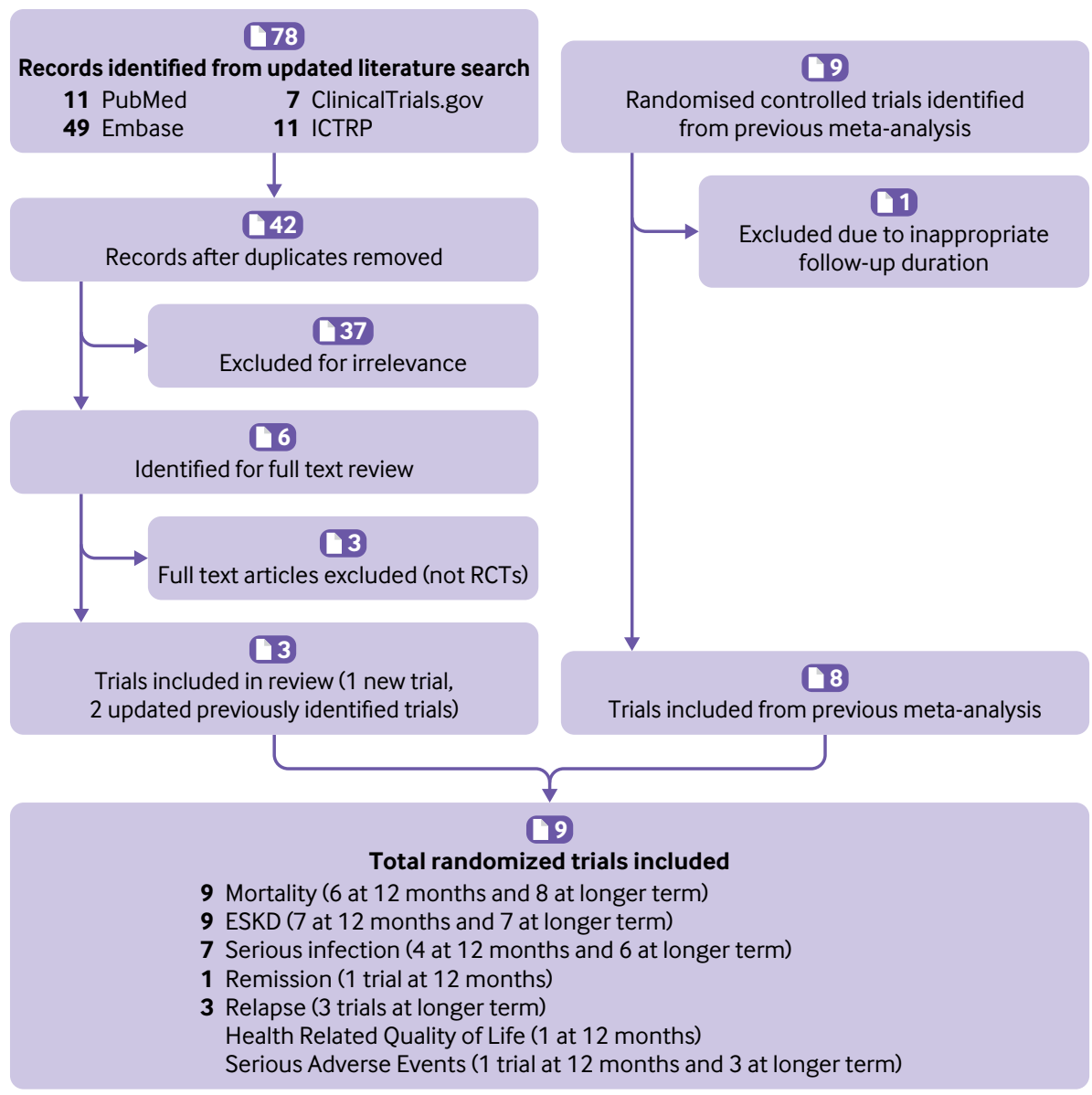

Fig 1 | Summary of eligible study identification.

time points less than 12 months after randomisation. ${ }^{20}$ Data from one trial was reported in two manuscripts (one short term and one long term follow-up), and data from two trials were reported as a single individual patient meta-analysis in a single manuscript. ${ }^{14} 1821$ Therefore, data from nine trials including 1060 patients with a median follow-up of three years were included (fig 1). ${ }^{31314181921-25}$ The characteristics of the studies are summarised in table 1.

\section{Risk of bias}

Table 2 presents the risk of bias for each trial. Five trials were at low risk of bias for random sequence generation and four were at low risk of bias for allocation concealment. No trials blinded participants, investigators, or outcome assessment, but-given the nature of the outcomes (death, ESKD, serious infections), which are unlikely to be affected by misascertainment, and for which no other therapies are known to be effective-the risk of bias was considered low. Eight $(89 \%)$ were considered at low risk of bias from incomplete data, and all were considered at low risk of bias from selective outcome reporting.

\section{Outcomes}

Table 3 summarises the findings of the main effects for all outcomes including absolute risk reductions. The results by subgroups are summarised in supplemental table 1 on bmj.com, and sensitivity analyses are provided in supplemental table 2.

\section{All-cause mortality}

Six trials including 967 patients reported all-cause mortality at 12 months, and eight trials including 1028 patients reported all-cause mortality at longer term follow-up. PLEX probably had little or no effect on allcause mortality at 12 months (relative risk 0.90 (95\% CI 0.64 to 1.27), moderate certainty) (fig 2) or longer term follow-up (RR 0.93 (0.73 to 1.19), moderate certainty) (supplemental fig 1). There was no evidence of subgroup effects by baseline kidney function on 12 month mortality $(\mathrm{P}=0.33)$ or longer term mortality $(\mathrm{P}=0.70)$ using data from three trials at each time point, each of which reported outcomes for both subgroups. These results were not materially different in sensitivity analyses using data from four trials for each group at each time point (supplementary table 1). Similarly, there was no evidence of heterogeneity on the basis of lung haemorrhage (RR 0.94 (0.36 to 2.45) in patients without lung haemorrhage $v$ RR 0.89 (0.01 to 92 ) in those with lung haemorrhage, interaction $\mathrm{P}=0.45$; supplementary table 1 ). Trial sequential analysis suggested it is unlikely further randomisation will result in proving at least a $20 \%$ risk reduction in 12 month mortality as the cumulative Z-curve lies fully within the futility boundary (supplemental fig 2). 


\begin{tabular}{|c|c|c|c|c|c|c|c|c|c|c|c|c|c|c|}
\hline \multirow[b]{3}{*}{ Study } & \multirow[b]{3}{*}{$\begin{array}{l}\text { Follow-up } \\
\text { (months) }\end{array}$} & \multirow{2}{*}{\multicolumn{3}{|c|}{ Plasma exchange }} & \multicolumn{5}{|c|}{ Participants } & \multirow{2}{*}{\multicolumn{2}{|c|}{$\begin{array}{l}\text { Baseline creatinine } \\
(\mu \mathrm{mol} / \mathrm{L})\end{array}$}} & \multirow{2}{*}{\multicolumn{2}{|c|}{$\begin{array}{l}\text { Baseline dialysis } \\
(\%)\end{array}$}} & \multirow[b]{3}{*}{$\begin{array}{l}\text { Lung } \\
\text { haem }\end{array}$} \\
\hline & & & & & \multirow[b]{2}{*}{ No } & \multicolumn{2}{|c|}{ Mean age (years) } & \multicolumn{2}{|c|}{ Female (\%) } & & & & & \\
\hline & & Method & $\begin{array}{l}\text { No of } \\
\text { treatments }\end{array}$ & $\begin{array}{l}\text { Volume/ } \\
\text { treatment }\end{array}$ & & wPLEX & Ctrl & PLEX & Ctrl & PLEX & Ctrl & PLEX & Ctrl & \\
\hline Rifle 1980 & 22 & Centrifuge & $\begin{array}{l}5 \text { in } 5 \text { days }+ \\
\text { additional for } \\
\text { non-response }\end{array}$ & $\begin{array}{l}1.5 \text { plasma } \\
\text { volumes }\end{array}$ & 14 & 41 & 52 & 50 & 25 & 893 & 1140 & 67 & 88 & No \\
\hline Mauri 1985 & 36 & $\begin{array}{l}\text { Centrifuge } \\
\text { and filter }\end{array}$ & $\begin{array}{l}6 \text { in } 12 \text { days + } \\
\text { additional for } \\
\text { non-response }\end{array}$ & $3.5 \mathrm{~L}$ & 22 & NR & NR & NR & NR & 1193 & 1158 & 50 & 50 & NR \\
\hline Pusey 1991 & 58 & Centrifuge & $\begin{array}{l}5 \text { in } 7 \text { days + } \\
\text { additional for } \\
\text { non-response }\end{array}$ & $4 \mathrm{~L}$ & 48 & 52 & 51 & 36 & 39 & 793 & 637 & 44 & 34 & Yes \\
\hline Cole 1992 & 12 & Centrifuge & $\geq 10$ in 16 days & 1 plasma volume & 32 & NR & NR & NR & NR & 634 & 769 & 25 & 43 & NR \\
\hline Guillevin 1997 & 12 & $\begin{array}{l}\text { Centrifuge } \\
\text { and filter }\end{array}$ & $\begin{array}{l}9 \text { or } 12 \text { at } 3 \\
\text { times/week }\end{array}$ & $60 \mathrm{~mL} / \mathrm{kg}$ & 32 & 47 & 62 & 47 & 38 & 439 & 287 & 32 & 15 & NR \\
\hline Zauner 2002 & 127 & NR & $\begin{array}{l}3+<9 \text { for non- } \\
\text { response }\end{array}$ & $40 \mathrm{~mL} / \mathrm{kg}$ & 39 & 55 & 56 & 29 & 22 & NR & NR & NR & NR & Yes \\
\hline $\begin{array}{l}\text { Jayne } 2007, \\
\text { Walsh } 2013\end{array}$ & 12,47 & $\begin{array}{l}\text { Centrifuge } \\
\text { and filter }\end{array}$ & 7 in 14 days & $60 \mathrm{~mL} / \mathrm{kg}$ & 137 & 67 & 66 & 41 & 36 & 701 & 732 & 67 & 71 & Yes \\
\hline Szpirt 2010 & 60 & Filter & $\begin{array}{l}6+3-6 \text { for } \\
\text { persistent ANCA }\end{array}$ & $4 \mathrm{~L}$ & 32 & 58 & 56 & 25 & 19 & 262 & 250 & 13 & 25 & Yes \\
\hline Walsh 2020 & 35 & $\begin{array}{l}\text { Centrifuge } \\
\text { and filter }\end{array}$ & 7 in 14 days & $60 \mathrm{~mL} / \mathrm{kg}$ & 704 & 63 & 64 & 42 & 45 & 327 & 336 & 19 & 21 & Yes \\
\hline
\end{tabular}

Lung haem $=$ Presence of lung haemorrhage at baseline. PLEX $=$ Plasma exchange. $\mathrm{Ctrl}=$ Control. NR $=$ Not reported.

End stage kidney disease (ESKD)

Seven trials including 999 patients reported ESKD at 12 months, and seven trials including 996 patients reported ESKD at longer term follow-up. PLEX probably reduced the risk of ESKD at 12 months (RR 0.62 (0.39 to 0.98)) (fig 3) and may affect longer term risk (RR 0.79 (0.58 to 1.08), low certainty) (supplemental fig 3 ). There was no evidence of subgroup effects based on kidney function at baseline at 12 months $(\mathrm{P}=0.55)$ or longer term followup $(\mathrm{P}=0.54)$ based on data from five trials reporting outcomes for both subgroups at 12 months and three trials at longer term follow-up (supplementary table 1). These results were not materially different when using data from all trials with available data for any subgroup. In trial sequential analysis, the cumulative information reached the adjusted monitoring boundary suggesting at least a 20\% risk reduction in ESKD by 12 months reliably exists (supplemental fig 4).

The estimated absolute risk reduction in ESKD for PLEX ranges from $0.08 \%$ (0.02\% to $0.12 \%)$ for those at the lowest risk (high certainty of no important effects), to $2.1 \%(0.6 \%$ to $3.1 \%)$ for those at low-moderate risk (low certainty), to $4.6 \%$ (1.2\% to $6.8 \%$ ) for those at moderate-high risk (moderate certainty), to $16.0 \%$ (4.2\% to $23.6 \%$ ) for those at high risk (high certainty of important effects).

\section{Serious infections}

Four trials including 908 patients reported serious infections at 12 months and six trials including 957 patients reported serious infections at longer term follow-up. PLEX increased the risk of serious infections at 12 months (RR 1.27 (1.08 to 1.49), moderate certainty) (fig 4) and may increase risk at longer term follow-up (RR 1.13 (1.03 to 1.24), low certainty) (supplemental fig 5). In trial sequential analysis, the cumulative information reached the adjusted monitoring boundary suggesting at least a 20\% risk increase in serious infections by 12 months reliably exists (supplemental fig 6).

The estimated absolute risk increase for serious infection with PLEX ranges from $2.7 \%$ (0.3\% to 5.6\%) for those at the lowest risk (moderate certainty), to $4.9 \%(0.5 \%$ to $10.1 \%)$ for those at low-moderate risk (moderate certainty), to $8.6 \%$ (1\% to $17.9 \%$ ) for those at moderate-high risk (moderate certainty), to $13.5 \%$ (1.5\% to $28.0 \%$ ) for those at high risk (moderate certainty).

\section{Remission}

No trials reported remission alone. One trial including 704 patients reported sustained remission as the composite of remission induced by six months and

\begin{tabular}{|c|c|c|c|c|c|c|}
\hline Study & $\begin{array}{l}\text { Random sequence } \\
\text { generation }\end{array}$ & $\begin{array}{l}\text { Allocation } \\
\text { concealment }\end{array}$ & $\begin{array}{l}\text { Blinding of participants } \\
\text { and investigators }\end{array}$ & $\begin{array}{l}\text { Blinding of outcome } \\
\text { assessment }\end{array}$ & $\begin{array}{l}\text { Incomplete } \\
\text { data }\end{array}$ & $\begin{array}{l}\text { Selective } \\
\text { reporting }\end{array}$ \\
\hline Rifle 1980 & Unclear & High & Low & Low & Low & Low \\
\hline Mauri 1985 & Unclear & High & Low & Low & Low & Low \\
\hline Pusey 1991 & Low & High & Low & Low & Low & Low \\
\hline Cole 1992 & Low & Low & Low & Low & High & Low \\
\hline Guillevin 1997 & Unclear & High & Low & Low & Low & Low \\
\hline Zauner 2002 & Unclear & High & Low & Low & Low & Low \\
\hline Jayne 2007, Walsh 2013 & Low & Low & Low & Low & Low & Low \\
\hline Szpirt 2011 & Low & Low & Low & Low & Low & Low \\
\hline Walsh 2020 & Low & Low & Low & Low & Low & Low \\
\hline
\end{tabular}




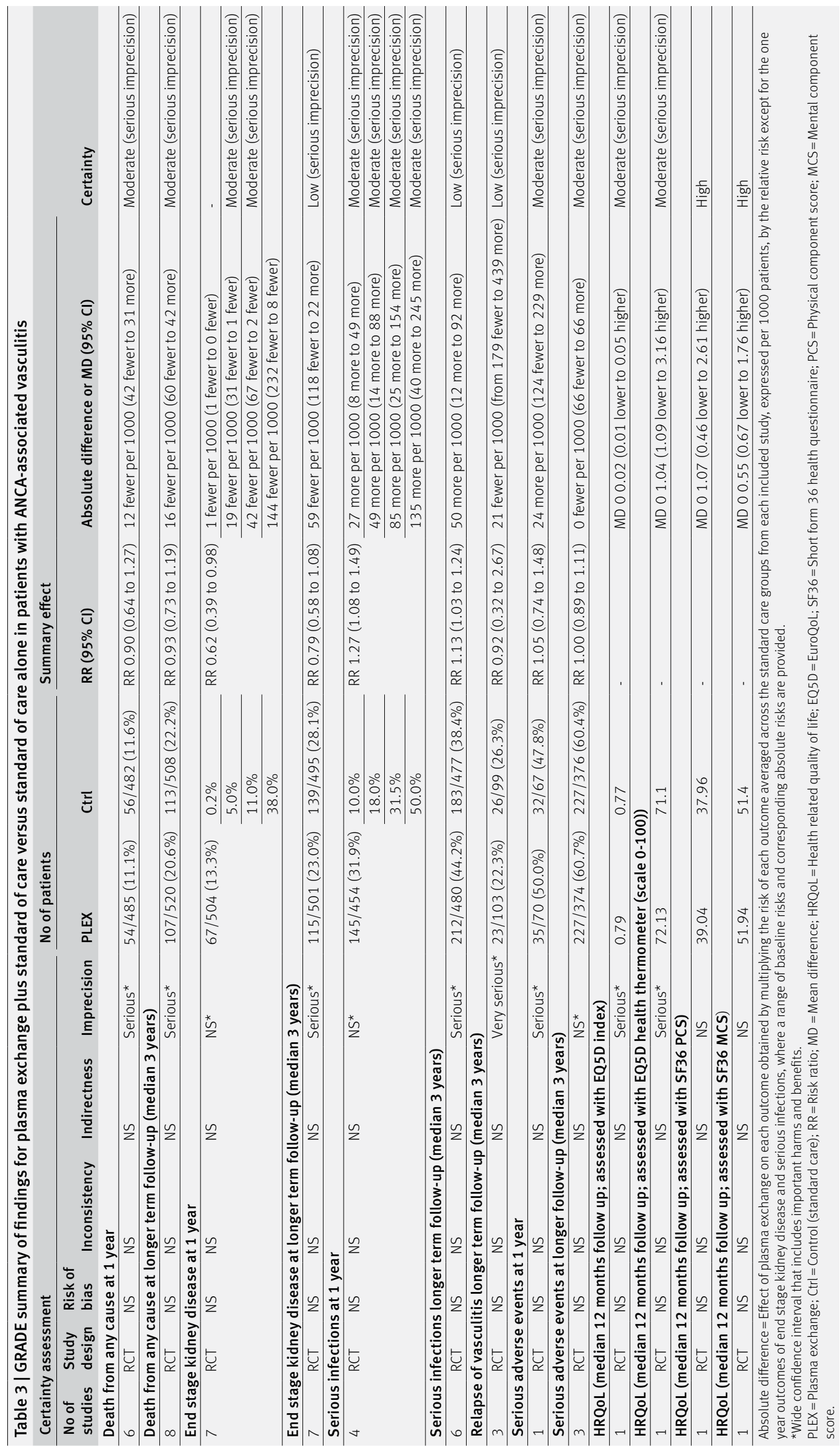

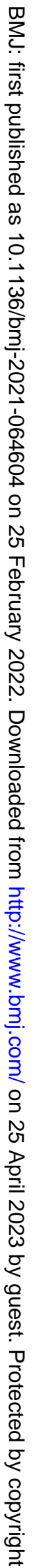




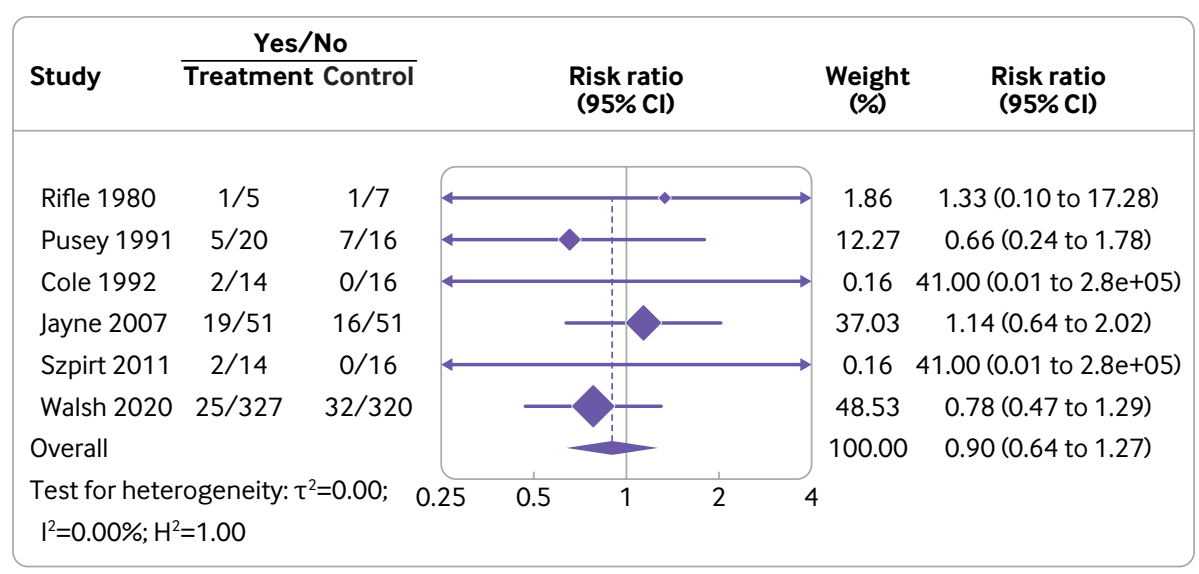

Fig 2 | Effect of plasma exchange on all-cause mortality within 12 months' follow-up in patients with anti-neutrophil cytoplasm antibody (ANCA)-associated vasculitis using the DerSimonian and Laird random effects model with KnappHartung standard error adjustment

sustained until at least 12 months and probably resulted in no appreciable difference with PLEX (RR 1.01 (0.89 to 1.15$)$, moderate certainty).

\section{Relapse}

Three trials including 202 patients reported relapse at longer term follow-up. PLEX may have little or no effect on the risk of relapse (RR 0.92 (0.32 to 2.67), low certainty).

Health related quality of life

Only one trial including 704 patients reported health related quality of life at 12 months, and no trials reported longer term effects on health related quality of life. No differences were seen at 12 months in any measure of health related quality of life (moderate to high certainty).

\section{Other serious adverse events}

One trial including 137 patients reported serious adverse events at 12 months, and three trials including
750 patients reported serious adverse events at longer follow-up. PLEX probably had no or little effect on serious adverse events at 12 months (RR 1.05 (0.74 to 1.48$)$, moderate certainty) or longer term follow-up (RR 1.00 (0.89 to 1.11), moderate certainty).

\section{Discussion}

\section{Principal findings}

This meta-analysis shows that PLEX results in reduced risk of end stage kidney disease (ESKD) and an increased risk of serious infections at 12 months in patients with anti-neutrophil cytoplasm antibody (ANCA)-associated vasculitis (AAV). Although the relative effects of PLEX on these outcomes were substantial, the baseline risks of ESKD and serious infections, and thus the absolute reduction in risks of ESKD and increases in serious infection with PLEX, vary substantially. The effects of PLEX on ESKD and serious infections at longer term follow-up were uncertain, but the point estimates were attenuated compared with 12 month outcomes. The effects of PLEX on all-cause mortality, regardless of

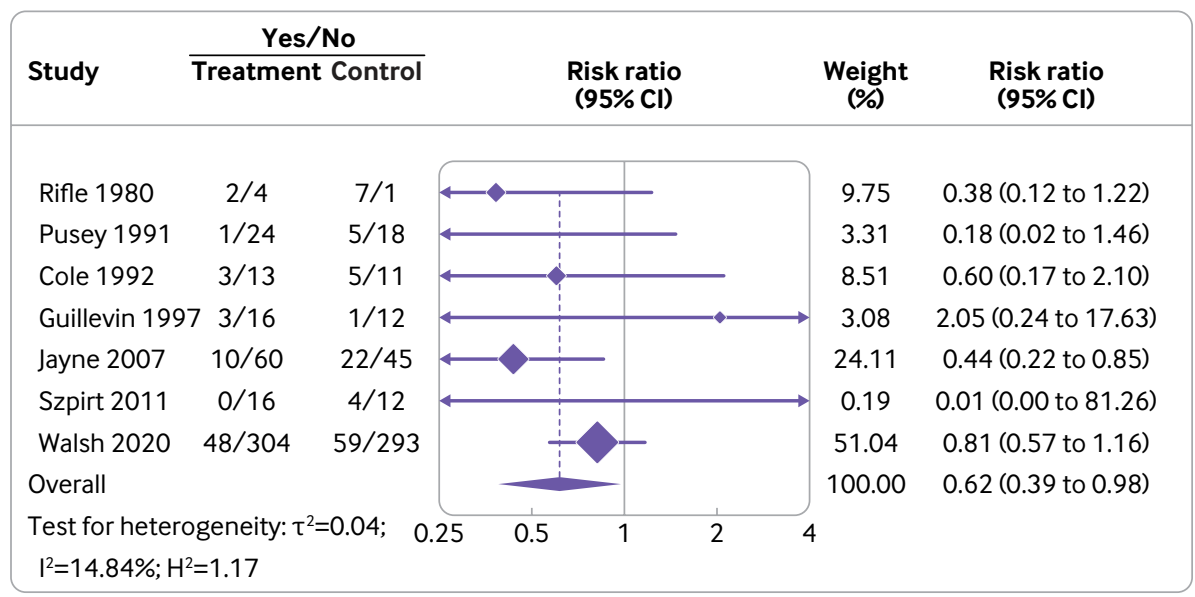

Fig 3 | Effect of plasma exchange on end stage kidney disease within 12 months' follow-up in patients with antineutrophil cytoplasm antibody (ANCA)-associated vasculitis using the DerSimonian and Laird random effects model with Knapp-Hartung standard error adjustment 


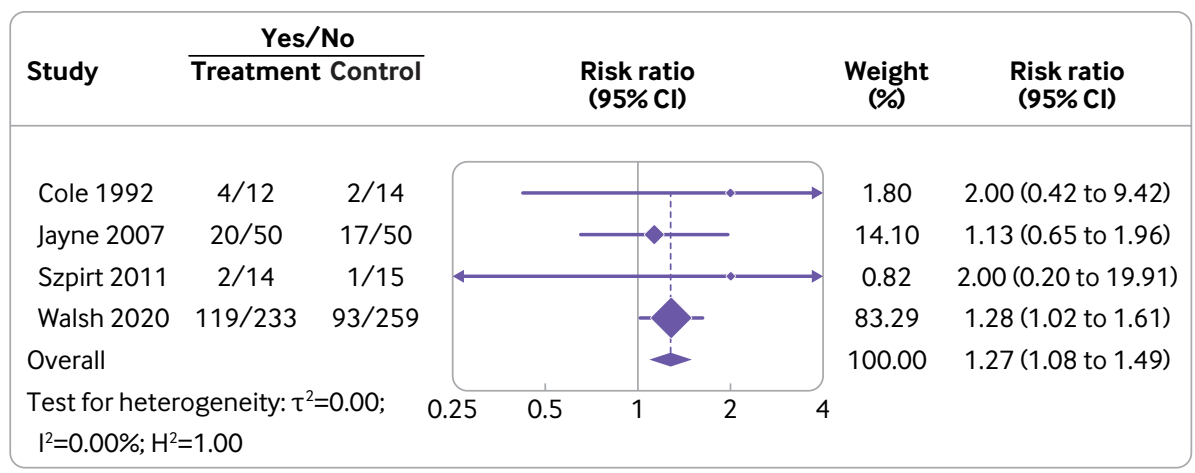

Fig 4 | Effect of plasma exchange on serious infection within 12 months' follow-up in patients with anti-neutrophil cytoplasm antibody (ANCA)-associated vasculitis using the DerSimonian and Laird random effects model with KnappHartung standard error adjustment

presence or absence of lung haemorrhage, and other outcomes were of low certainty both at 12 months and at longer term follow-up.

\section{Strengths and limitations of this study}

The strengths of this review include a comprehensive search, the reporting of important outcomes, the assessment of optimal information size with trial sequential analysis and the GRADE approach to report certainty of evidence. ${ }^{26}$ In addition, we reported the absolute effects across the broad range of risks with which patients with this disease present.

The limitations of this review include the relative paucity of RCT data in general and, in particular, the paucity of data informing potential subgroup effects. Despite this, there was sufficient evidence to be moderately certain an important effect exists for the outcome of ESKD. With regard to subgroup effects, the available data do not suggest heterogeneity of effects across subgroups, and there are few biological explanations for why a subgroup effect might exist; our panel thus concluded subgroup effects are unlikely to exist. A further limitation was the moderate degree of clinical heterogeneity across studies that included patients with potentially different diagnoses, different regimens of PLEX, and different therapies representing standard care. Finding a robust effect on ESKD may be considered reassuring since the clinical heterogeneity might be expected to bias the result towards the null. Also, most of the data come from the most recent trials, in which the diagnoses and treatment regimens were more homogeneous. A final limitation is the imprecision with which baseline infection risk can be calculated and mapped to risk of ESKD. We can, however, be confident that risk of serious infections is higher in those with worse kidney function both in AAV and other acute and chronic conditions. ${ }^{1272829}$

\section{Comparison with other studies}

There is perhaps a counter-intuitive difference between the result of this review and PEXIVAS, the largest trial of PLEX in AAV. PEXIVAS failed to show a difference between PLEX and control for both the primary composite outcome of ESKD or all-cause mortality and for each of the components of the primary outcome separately. PEXIVAS, however, was designed to detect an approximate 34\% relative risk reduction in the composite outcome of ESKD or all-cause mortality on the basis of an estimated 164 primary outcome events. $^{30}$ This review found a $42 \%$ relative risk reduction in ESKD at 12 months on the basis of 167 outcome events (117 of which were from PEXIVAS) but uncertain effects on ESKD at longer term follow-up or on all-cause mortality at any time.

This meta-analysis demonstrates the unexpected, differential effects of PLEX on ESKD compared with all-cause mortality, which dramatically reduced the power of PEXIVAS to reliably estimate the benefits of PLEX using a composite outcome. Additionally, the discordant effects of PLEX on ESKD compared with allcause mortality noted in previous trials and reviews, and attributed to random error, are now more precisely estimated and a potential explanation has become evident-a previously unidentified, concomitant increase in the risk of serious infection. ${ }^{5}$ The increased risk of serious infection is not, to our knowledge, reliably demonstrated in other clinical areas. $^{31}$ This may be due to the larger number of patients randomised and relatively high baseline rates of infection in AAV compared with other conditions resulting in greater statistical power, or potentially an interaction with the concomitant glucocorticoids and immunosuppressive therapies used to treat AAV.

This systematic review included substantially more data than our previous review and used more granular data than other systematic reviews. ${ }^{52}$ Accordingly, the estimates from this review are more refined than those of previous reviews and, compared with our previous meta-analysis, the current review reliably concludes that PLEX reduces the risk of ESKD within 12 months but is responsible for a concomitant increase in the risk of serious infections not previously appreciated. This is an important finding as it may explain the lack of effect of PLEX on mortality despite the large effect on ESKD. We provide absolute risk reductions and increases for important outcomes that will help healthcare providers, patients, and family members engage in shared decision making. ${ }^{33}$ 


\section{Implications of the study}

This systematic review provides information regarding the benefits and harms of PLEX in AAV necessary for decision makers. The review identifies moderate certainty evidence that PLEX reduces the risk of ESKD within one year. It is uncommon to generate this degree of certainty for such an impactful outcome in a rare disease. The available data further suggest that the effects of PLEX on both ESKD and serious infections diminish over time. The complicated trade-off between ESKD and serious infections that changes over time informs shared decision making. Furthermore, since PLEX is an invasive, expensive therapy that is not ubiquitously available, understanding these effects across the range of baseline risks is important in understanding the cost-effectiveness of PLEX in AAV (very low in the lowest risk patients, substantial in the higher risk patients).

\section{AUTHOR AFFILIATIONS}

${ }^{1}$ Department of Medicine, McMaster University, Hamilton, Canada

${ }^{2}$ Department of Health Research Methods, Evidence and Impact, McMaster University, Hamilton, Canada

${ }^{3}$ Population Health Research Institute, Hamilton Health Sciences / McMaster University, Hamilton, Canada

${ }^{4}$ Department of Medicine, University of Alberta, Edmonton, Canada

${ }^{5}$ Department of Pharmacy/Evidence-based Pharmacy Centre, West China Second University Hospital, Sichuan University, Chengdu, China

${ }^{6}$ Division of Rheumatology, Department of Medicine and Division of Clinical Epidemiology, Department of Biostatistics, Epidemiology and Informatics, University of Pennsylvania, Philadelphia, USA

${ }^{7}$ Department of Immunology and Inflammation, Imperial College London, London, UK

${ }^{8}$ Royal Adelaide Hospital, Adelaide, Australia

${ }^{9}$ University of Adelaide, Adelaide, Australia

${ }^{10}$ Rigshospitalet University Hospital, Department of Nephrology, Copenhagen, Denmark

${ }^{11}$ Institute for Advancement of Clinical and Translational Science, Kyoto University Hospital, Kyoto, Japan

${ }^{12}$ Clinical and Translational Research Center, University Hospital, Kyoto Prefectural University of Medicine, Kyoto, Japan

${ }^{13}$ Department of Medicine, University of Cambridge, United Kingdom

Contributors: MW, PM, and DJ conceived of the study idea. MW performed the literature search. MW and DTC performed the screening, data abstraction, and risk of bias assessments. MW performed the data analysis. MW, DTC, DJ, PM, LZ, and GHG interpreted the data. MW, DTC, LZ, and GHG performed the certainty assessment. MW, GHG, LZ, and DTC drafted the manuscript. All authors critically revised the manuscript and approved the final version of the manuscript. MW had full access to the data in the study and takes responsibility for the integrity of the data and the accuracy of the data analysis. MW is the guarantor. The corresponding author attests that all listed authors meet authorship criteria and that no others meeting the criteria have been omitted.

Funding: This research received no specific grant from any funding agency in the public, commercial or not-for-profit sectors. MW is funded by a McMaster University Department of Medicine Clive Kearon Mid-Career Award. DJ is supported by the Cambridge Biomedical Centre.

Competing interests: All authors have completed the ICMJE uniform disclosure form at www.icmje.org/coi_disclosure.pdf and declare: no support from any organisation for the submitted work; PAM reports receiving funds for consulting and research support from AbbVie, AstraZeneca, Boeringher-Ingelheim, Bristol-Myers Squibb, ChemoCentryx, Forbius, Genentech/Roche, Genzyme/ Sanofi, GlaxoSmithKline, Inflarx, and Takeda and consulting for CSL Behring, Dynacure, EMDSerono, Jannsen, Kliniksa, Kyverna, Magenta, MiroBio, Neutrolis, Novartis, Pfizer, Sparrow, and Talaris and royalties from UpToDate. DRWJ reports consulting fees from AstraZeneca, Chemocentryx and Genentech and grant support paid to the University of Cambridge and consulting fees from GlaxoSmithKline; no other relationships or activities that could appear to have influenced the submitted work.

Data sharing: No additional data are available.

Transparency: The lead author affirms that the manuscript is an honest, accurate, and transparent account of the study being reported; that no important aspects of the study have been omitted; and that any discrepancies from the study as planned have been explained

Dissemination to participants and related patient and public communities: We plan to disseminate the results through publications, conference presentations, and social media to international stakeholders, including patient led organisations, and healthcare decision makers who use or generate evidence based on routinely collected data.

This is an Open Access article distributed in accordance with the Creative Commons Attribution Non Commercial (CC BY-NC 4.0) license, which permits others to distribute, remix, adapt, build upon this work non-commercially, and license their derivative works on different terms, provided the original work is properly cited and the use is noncommercial. See: http://creativecommons.org/licenses/ by-nc/4.0/.

1 Little MA, Nightingale P, Verburgh CA, et al, European Vasculitis Study (EUVAS) Group. Early mortality in systemic vasculitis: relative contribution of adverse events and active vasculitis. Ann Rheum Dis 2010;69:1036-43. doi:10.1136/ard.2009.109389

2 Flossmann O, Berden A, de Groot K, et al, European Vasculitis Study Group. Long-term patient survival in ANCA-associated vasculitis. Ann Rheum Dis 2011;70:488-94. doi:10.1136/ard.2010.137778

3 Walsh M, Merkel PA, Peh CA, et al, PEXIVAS Investigators. Plasma exchange and glucocorticoids in severe ANCA-associated vasculitis. N Engl J Med 2020;382:622-31. doi:10.1056/NEJMoa1803537

4 Zeng L, Walsh M, Guyatt G, et al. Plasma exchange and glucocorticoid dosing for patients with ANCA-associated vasculitis: a clinical practice guideline. BMJ 2022;376:e064597.

5 Walsh M, Catapano F, Szpirt W, et al. Plasma exchange for renal vasculitis and idiopathic rapidly progressive glomerulonephritis: a meta-analysis. Am J Kidney Dis 2011;57:566-74. doi:10.1053/j. ajkd.2010.10.049

6 Jennette JC, Falk RJ, Bacon PA, et al. 2012 revised International Chapel Hill Consensus Conference Nomenclature of Vasculitides. Arthritis Rheum 2013;65:1-11. doi:10.1002/art.37715

7 Higgins JP, Thomas J, Chandler J, et al, eds. Cochrane handbook for systematic reviews of interventions. John Wiley \& Sons, 2019. doi:10.1002/9781119536604

8 Hultcrantz M, Rind D, AkI EA et al. The GRADE Working Group clarifies the construct of certainty of evidence. / Clin Epidemiol 2017;87:4-13. doi:10.1016/j.jclinepi.2017.05.006

9 DerSimonian R, Laird N. Meta-analysis in clinical trials. Control Clin Trials 1986:7:177-88 doi:10.1016/0197-2456(86)90046-2

10 Hartung J, Knapp G. A refined method for the meta-analysis of controlled clinical trials with binary outcome. Stat Med 2001:20:3875-89. doi:10.1002/sim.1009

11 Agresti A. An introduction to categorical data analysis. John Wiley \& Sons, 2018

12 Sweeting MJ, Sutton AJ, Lambert PC. What to add to nothing? Use and avoidance of continuity corrections in meta-analysis of sparse data. Stat Med 2004;23:1351-75. doi:10.1002/sim.1761

13 Pusey CD, Rees AJ, Evans DJ, Peters DK, Lockwood CM. Plasma exchange in focal necrotizing glomerulonephritis without anti-GBM antibodies. Kidney Int 1991;40:757-63. doi:10.1038/ki.1991.272

14 Jayne DR, Gaskin G, Rasmussen N, et al, European Vasculitis Study Group. Randomized trial of plasma exchange or high-dosage methylprednisolone as adjunctive therapy for severe renal vasculitis. J Am Soc Nephrol 2007;18:2180-8. doi:10.1681/ASN.2007010090

15 Balogun RA, Sanchez AP, Klingel R, et al. Update to the ASFA guidelines on the use of therapeutic apheresis in ANCA-associated vasculitis. J Clin Apher 2020;35:493-9. doi:10.1002/jca.21820

16 Wetterslev J, Thorlund K, Brok J, Gluud C. Trial sequential analysis may establish when firm evidence is reached in cumulative meta-analysis. J Clin Epidemiol 2008;61:64-75. doi:10.1016/j. jclinepi.2007.03.013

17 Walsh M. Predicting the 1-year risk of kidney failure in ANCA associated vasculitis. BMC Medicine (forthcoming).

18 Walsh M, Casian A, Flossmann O, et al, European Vasculitis Study Group (EUVAS). Long-term follow-up of patients with severe ANCA associated vasculitis comparing plasma exchange to intravenous methylprednisolone treatment is unclear. Kidney Int 2013;84:397402. doi:10.1038/ki.2013.131 
19 Szpirt WM, Heaf JG, Petersen J. Plasma exchange for induction and cyclosporine A for maintenance of remission in Wegener's granulomatosis--a clinical randomized controlled trial. Nephrol Dial Transplant 2011;26:206-13. doi:10.1093/ndt/gfq360

20 Glöckner WM, Sieberth HG, Wichmann HE, et al. Plasma exchange and immunosuppression in rapidly progressive glomerulonephritis: a controlled, multi-center study. Clin Nephrol 1988;29:1-8

21 Guillevin L, Cevallos R, Durand-Gasselin B, Lhote F, Jarrousse B, Callard P. Treatment of glomerulonephritis in microscopic polyangiitis and Churg-Strauss syndrome. Indications of plasma exchanges, meta-analysis of 2 randomized studies on 140 patients, 32 with glomerulonephritis. Ann Med Interne (Paris) 1997;148:198-204.

22 Rifle G, Chalopin JM, Zech P, et al. Treatment of idiopathic acute crescentic glomerulonephritis by immunodepression and plasmaexchanges. A prospective randomised study. Proc Eur Dial Transplant Assoc 1981;18:493-502.

23 Mauri J, Gonzalez M, Poveda R, et al. Therapeutic plasma exchange in the treatment of rapidly progressive glomerulonephritis. Plasma Ther Transfus Technol 1985;6:587-91.

24 Cole E, Cattran D, Magil A, et al, The Canadian Apheresis Study Group. A prospective randomized trial of plasma exchange as additive therapy in idiopathic crescentic glomerulonephritis. Am J Kidney Dis 1992;20:261-9. doi:10.1016/S02726386(12)80699-8

25 Zäuner I, Bach D, Braun N. Predictive value of initial histology and effect of plasmapheresis on long-term prognosis of rapidly progressive glomerulonephritis. Am J Kidney Dis 2002;39:28-35. doi:10.1053/ajkd.2002.29874

26 Brozek JL, Akl EA, Alonso-Coello P, et al, GRADE Working Group. Grading quality of evidence and strength of recommendations in clinical practice guidelines. Part 1 of 3 . An overview of the GRADE approach and grading quality of evidence about interventions. Allergy 2009;64:669-77. doi:10.1111/j.1398-9995.2009.01973.x
27 Ishigami J, Grams ME, Chang AR, Carrero JJ, Coresh J, Matsushita K. CKD and risk for hospitalization with infection: the Atherosclerosis Risk in Communities (ARIC) Study. Am J Kidney Dis 2017;69:752-61. doi:10.1053/j.ajkd.2016.09.018

28 Ishigami J, Trevisan M, Xu H, Coresh J, Matsushita K, Carrero JJ. Estimated GFR and hospital-acquired infections following major surgery. Am Kidney Dis 2019;73:11-20. doi:10.1053/j.ajkd.2018.06.029

$29 \mathrm{Xu} \mathrm{H}$, Gasparini A, Ishigami J, et al. eGFR and the risk of communityacquired infections. Clin J Am Soc Nephrol 2017;12:1399-408. doi:10.2215/CJN.00250117

30 Walsh M, Merkel PA, Peh CA, et al, PEXIVAS Investigators. Plasma exchange and glucocorticoid dosing in the treatment of antineutrophil cytoplasm antibody associated vasculitis (PEXIVAS): protocol for a randomized controlled trial. Trials 2013;14:73. doi:10.1186/1745-6215-14-73

31 Chevret S, Hughes RA, Annane D. Plasma exchange for GuillainBarré syndrome. Cochrane Database Syst Rev 2017;2:CD001798. doi:10.1002/14651858.CD001798.pub3

32 Walters GD, Willis NS, Cooper TE, Craig IC. Interventions for renal vasculitis in adults. Cochrane Database Syst Rev 2020;1:CD003232. doi:10.1002/14651858.CD003232.pub4

33 Hembroff LA, Holmes-Rovner M, Wills CE. Treatment decision-making and the form of risk communication: results of a factorial survey. BMC Med Inform Decis Mak 2004;4:20. doi:10.1186/1472-6947-4-20

Supplementary material: Details of study collaborators; supplementary tables 1 (main and subgroup effect estimates) and 2 (sensitivity analyses of dichotomous outcomes); and supplementary figs 1-6 (outcomes at longer term follow-up, and trial sequential analysis for outcomes within 12 months) 